\title{
Theileria-Induced Leukocyte Transformation: A Parasite Model for the Study of Lympho Proliferation
}

\author{
Aquil Mohmad $^{1 *}$, Bilal Ahmad Malla ${ }^{2}$, Ishfaq Maqbool ${ }^{3}$, \\ Zahoor Ahmad War ${ }^{4}$, Irshad Ahmad Para ${ }^{5}$ and Sheikh Firdous Ahmad ${ }^{6}$
}

${ }^{1}$ Division of Parasitology, ${ }^{2}$ Division of Veterinary Public Health, ${ }^{4}$ Division of Veterinary Pathology,

${ }^{5}$ Division of Physiology and climatology, Indian Veterinary Research Institute, Izatnagar, Bareilly-243122, UP, India

${ }^{3}$ Department of Veterinary Parasitology, College of Veterinary Science, Guru Angad Dev Veterinary and Animal Sciences University, Ludhiana-141004, Punjab, India

${ }^{6}$ Division of Animal Genetics, ICAR-Indian Veterinary Research Institute, Izatnagar

Bareilly 243122 UP India.

*Corresponding author

A B S T R A C T

The genus Theileria includes tick-transmitted Apicomplexan parasites of ruminants with substantial economic impact in endemic countries. Amongst the Apicomplexa phylum, Theileria is the only genus which transforms its mammalian host cells. Theileria parva and

\section{Keywords}

Theileria parva and Theileria annulata, Exotic (taurine), P 53, immortalization, NF-kB

Article Info

Accepted:

22 October 2018 Available Online: 10 November 2018
Theileria annulata infect leukocytes where they induce phenotypes that are shared with some cancers such as immortalization, dissemination and hyper proliferation. Transformation of lymphocytes is induced during the schizont stage. The mechanism of host cell transformation induces constitutive activation of bovine transcription factors (NF$\mathrm{kB}$ ) and associated signal transduction pathways. P53 plays an important role in proliferation and apoptosis and thus represents a key molecule in tumor formation. Moreover T. annulata schizonts induce a Warburg effect in host cells by a shift in ATP generation from predominantly oxidative phosphorylation to glycolysis and Theileria parasites avoids autophagic clearance by directly blocking autophagy. Theileria parasites secrete a protein, prolyl isomerase for maintaining the transformation of host cells. The host cell transformation by Theileria parasites and the comparison between cancer biology and host-Theileria interactions can help in revealing chemotherapeutic targets. This review is about host cell manipulation induced by Theileria parasites and the phenotypes, Theileria infected cells share with many cancers.

\section{Introduction}

Tropical theileriosis and East Coast Fever are tick-borne diseases of cattle caused by the protozoan parasites Theileria annulata and
Theileria parva, respectively. They cause lymphoproliferative disorders of cattle (Irvin and Morrison, 1987). Exotic (taurine) breeds of cattle and their crosses are more susceptible to the disease and its clinical course may 
prove fatal. However, Indian breeds of cattle are relatively resistant and act as reservoir hosts. Pathology of the disease is associated with the presence of intra-macrophage stage of the parasite (schizont) but the exact mechanisms remain unclear (Preston et al., 1999; Glass, 2001; Preston, 2001). Theileria parasites enter the bovine hosts during tick feeding as sporozoites, which rapidly invade mononuclear leukocytes in the lymph glands nearest to tick bite. Unlike many apicomplexans, Theileria resides in the host cytosol instead of inside a parasitophorous vacuole and during host cell mitosis, the schizonts bind to the host mitotic spindle, ensuring segregation into both daughter cells with great efficiency to maintain the infection rate. Macroschizonts develop further into microschizonts and ultimately into merozoites, which are released from the leukocyte. The merozoites invade erythrocytes and develop into piroplasms (Tait and Hall, 1990). T. annulata infects monocytes/macrophages and B cells of bovine, ovine, and caprine origin (Dobbelaere and Heussler, 1999; Schnittger, 2000), but only bovine leukocytes are transformed. The macroschizont stage is largely responsible for the pathology of Theileria infections (Hooshmand-Rad, 1976). The intracellular multinucleated parasite induces the proliferation of its host cell, leading to the clonal expansion of the parasitized cell population (Hulliger et al., 1964; Nichani et al., 1999). The synchrony between host cell and parasite cell cycles, together with the close association of the macroschizont with the host cell spindle microtubules, ensures that each daughter cell inherits a parasite at the completion of mitosis (Hulliger et al., 1964). The conversion of Theileria schizont-infected cells into immortal cell lines depends on acquired characteristics that are remarkably similar to those exhibited by some cancerous cells, such as immune evasion and resistance to apoptosis, and possibly provides a better understanding of the molecular interactions underlying these phenotypes (Hulliger et al., 1966; SchmuckliMaurer et al., 2008; Shiels et al., 1992). Like Plasmodia, in their mammalian hosts, Theileria parasites are haploid. Diploid parasites and genetic recombination only take place in the insect vector that are ticksHyalomma for T. annulata and Rhipicephalus for T. parva. It is the distribution of the two tick species that determines the distribution of the two diseases.

\section{Host cell transformation}

Theileria-induced transformation leads to activation of nuclear factor kappa-light-chainenhancer of activated B cells (NF-kB)dependent proliferative signaling. Transformation of $T$. annulata and T. parvainfected leucocytes induce constitutive activation of bovine transcription factors and associated signal transduction pathways (Shiels et al., 2006). Theileria schizonts constitutively activate the IKK complex on their cell surface by trans-autophosphorylation and IkB kinase (IKK) complex activates NFkB (Hayashida et al., 2013). NF-kB leads into the nucleus and transcriptional activity starts and during host cell mitosis, the schizonts bind to the host mitotic spindle ensuring segregation into both daughter cells with great efficiency to maintain the infection rate (Von Schubert et al., 2010). NFkB (nuclear factor, p50 and p65) is an important transcription factor (a protein that interacts with the promoter of genes and stimulates gene expression). NFkB is bound by $\mathrm{IkB}$ (its inhibitor) which retains it in the cytoplasm and keeps it inactive and Phosphorylation followed by ubiquitinylation and degradation of IkB leads to import into the nucleus and transcriptional activity. Theileria interferes with this pathway by causing the destruction of $\mathrm{IkB}$ and it is a major player in the stimulation and clonal expansion of lymphocytes (Von Schubert et al., 2010). 
While bovine leukocytes transformed by $T$. parva, but not by $T$. annulata can produce interferon $\mathrm{g}$ (IFN-g) and interleukin 2 (IL-2), both produce and respond to tumor necrosis factor a (TNF-a). However, it has been suggested that some Theileria-transformed cell lines may grow independently of growth factors (Reddel, 2000; Ahmed et al., 1987).

\section{Immune evasion in Theileria-infected cells}

Immune evasion may involve modulation of the host immune response. Even though possible molecular mechanisms are unclear, some patterns are emerging that inflammatory cytokine production (e.g., IFN-g) is somehow delayed by the parasite in vivo until after schizont development, when signaling is not as effective (Campbel et al., 1999). This may be one reason why $T$. annulata is not cleared by a Th1 response, even though this is typically the case for other macrophageresident protozoan infections. $T$. annulatainfected bovine macrophages down regulate some macrophage markers and lose functions such as Fc- mediated phagocytosis and the production of antimicrobial molecules including nitric oxide and TNF-a (Jensen, K. et al., (2009). The inflammatory cytokine production (e.g., IFN-g) is delayed by the parasite until after schizont development when signaling is not as effective and Theileria infected activated $\mathrm{T}$ cells migrate from the lymph nodes to the efferent lymph downregulate CD2 a crucial adhesion molecule for cytolytic activity. Parasitetransformed cells cause marked proliferative response of uninfected lymphocytes this phenomenon is called the autologous Theileria mixed-lymphocyte reaction (Nichani et al., 1999; Campbel et al., 1999). T. parva-infected lymphocytes can also upregulate several immunoregulatory molecules, including IFN-g and IL- 2, both of which improve the transformation efficiency of host lymphocytes (Shayan et al., 1999). Because MHC I is essential for $T$. parva invasion of bovine lymphocytes and IFN-g is known to upregulate MHC I (Shaw et al., 1995; Zhou et al., 2009), the expression of IFN-Y is an apparent mechanism by which the parasite can increase the susceptibility of circulating lymphocytes to infection, and proliferative cytokines likely aid the division of parasitized cells. However, cytokine profiles often vary among $T$. parva-infected bovine $\mathrm{T}$ cell clones.

Pathogens and tumors have also been known to use other immune evasion strategies, such as sequestration, dormancy, antigenic variation and failure of antigen display. Investigations into these potential mechanisms of immune evasion in cattle and buffalo could lead to insights that are crucial for the improvement of vaccine regimens.

\section{Role of p53 gene in survival of Transformed lymphocyte cell}

P53 is important protein involved in proliferation and apoptosis and thus represents a key molecule in tumor formation. It arrests cell division and to initiate apoptosis in the case of cell stress and DNA damage, protecting the integrity of the genome. The central function of p53 is preventing the onset of cancer (Levine, 1997). Theileria schizonts induce anti-apoptotic proteins such as cellular FLICE-like inhibitory protein (cFLIP) and cellular inhibitor of apoptosis proteins (cIAPs) by activating host IKK complexes and upregulating or maintaining high c-MYC expression. T. annulata schizont leads to cytoplasmic sequestration of the majority of host cell p53 resulting in the inhibition of p53mediated apoptosis and promotion of host cell survival. Elimination of the parasite leads to nuclear translocation of p53 resulting in the upregulation of the proapoptotic proteins and the downregulation of an anti-apoptotic protein (D Haller et al., 2010) (Fig. 1 and 2). 
Fig.1 (NF-kB)-dependent proliferative signaling

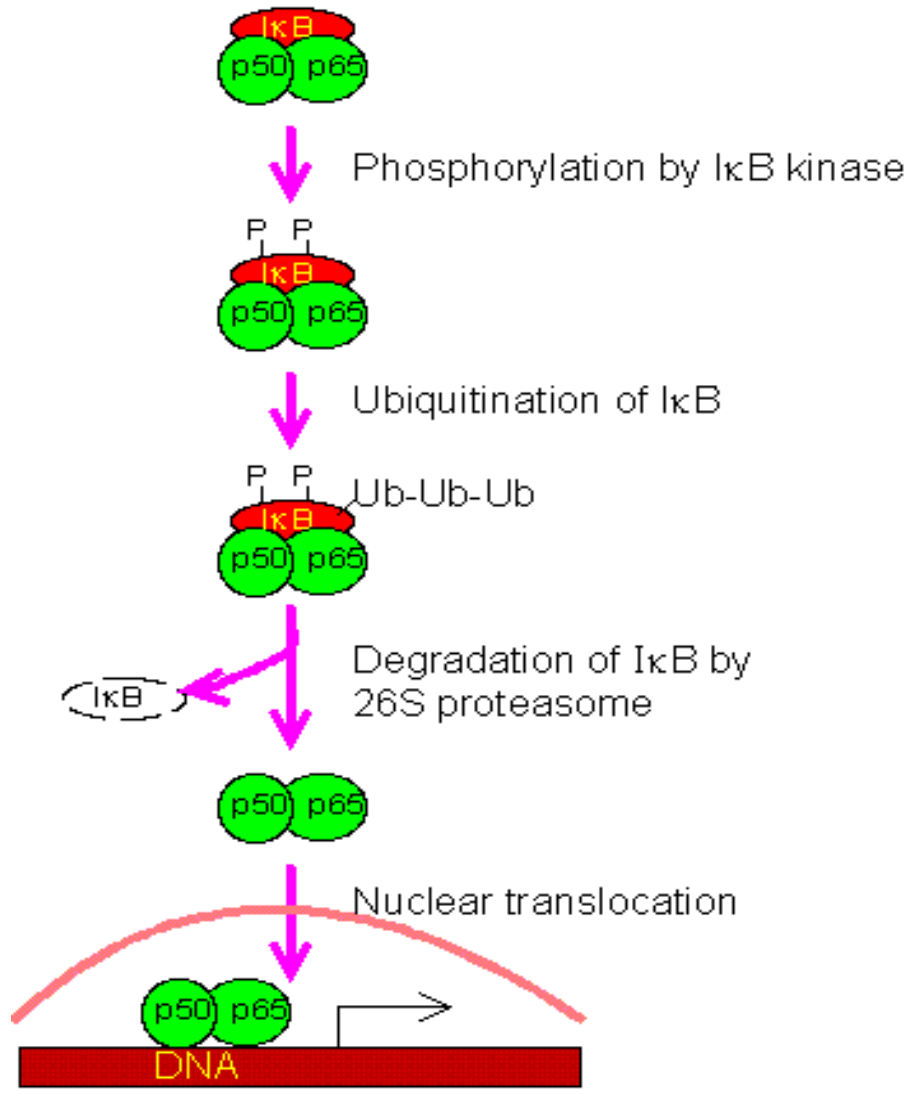

Fig.2 Parasite coordinated NF-KB activation

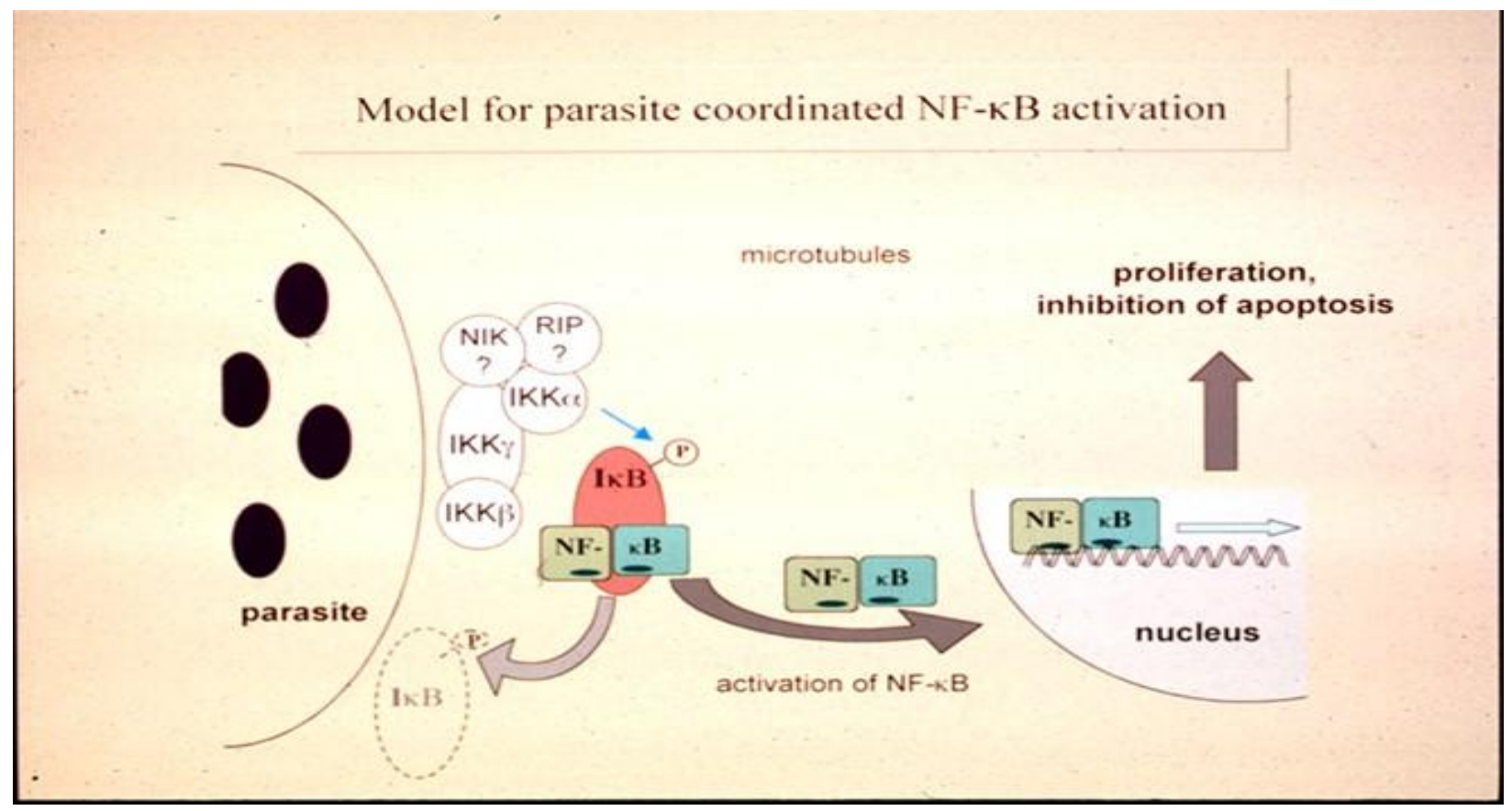




\section{Deregulation of cellular energetics}

Theileria parasites take nutrients from cytosol of cell and the acquisition of metabolites from the cytosol depletes nutrients from the host. Moreover, T. annulata schizonts induce a Warburg effect in host cells defined by a shift in ATP generation from predominantly oxidative phosphorylation to glycolysis. This metabolic switch is associated with a deregulation in the concentration of reactive oxygen species (ROS) and activation of the protein hypoxia-inducible factor 1a (HIF-1a) (Metheni et al., 2015). Cells can survive metabolic stress by the induction of autophagy that will result in the clearance of Theileria parasites from the host cytosol, because defects in autophagy have been associated with increased tumorigenesis in some cancers, determining how Theileria parasites avoid autophagic clearance, perhaps by directly blocking autophagy, without inducing metabolic stress-induced cell death could provide key insights into the coregulation of autophagy and metabolism (Duszenko et al., 2011).

\section{Metastasis by Theileria spp.}

Sporozoite micronemes secretions causes hydrolysis of lipids in plasma membrane and the sporozoite surface firmly attaches lymphocyte plasma membrane. Lymphocyte plasma membrane lipids and sporozoite rhoptries derived lipids helps in formation of parasitophorous vesicle around internalized sporozoite and sporozoites escape from vesicle by secretion of phospholipases by sporozoites. $\quad T$. annulata-infected macrophages invade tissues via an amoeboid invasion mechanism, for which matrix metalloproteinase 9 (MMP-9), transforming growth factor b (TGF-b), and TNF-a are essential (Ma and Baumgartner, 2014). Theileria-transformed host cells proliferate and disseminate into various organs causing lymph node swelling and Theileria parasites have been shown to have a close association with host microtubules which play a crucial role in metastasis. $T$. annulata recruits endbinding protein 1 to its cell surface by interactions with $T$. annulata polymorphic piroplasm antigen p104 and TaSE (Woods et al., 2013).

\section{Differences between theileria-infected cells and cancer cells}

Theileria transformed bovine cell proliferation may lack some of the characteristics of cancer cells and two such phenotypes are the evasion of growth suppression and a breakdown in genomic integrity. Most somatic cells stop proliferating at a specific density as a result of interactions with other cells known as contact inhibition. Cancer cells must overcome this barrier for proliferation and to grow. Theileria transformed lymphocytes require contact with other infected or uninfected cells to proliferate. When cells are cured from parasite infection they die (by apoptosis -- this suicide response is usually suppressed in cancer cells) Seluanov et al., 2009; Dobbelaere et al., (1991). Although there are no reports of genomic instability in Theileria-transformed cells, there is evidence that Theileria parasites and other Apicomplexans such as Cryptosporidium and Toxoplasma affect host DNA integrity (Haller et al., 2010). Theileria parasites have been shown to sequester p53 on their surface, in the host cytosol, presumably preventing it from executing its role in maintaining genomic stability.With all of the hallmarks that Theileria transformation has in common with many kinds of cancers. Cancer is a genetic disease induce genomic mutations in their host and is irreversible, but there are no reports of genomic instability in Theileria-transformed cells as treatment reverses the effect of transformed cells and these cells dies by apoptosis (Vogelstein et al., 2013; Weitzman, 2014). 


\section{Parasite molecules required for the cancer-} like phenotypes

A question persists in the study of Theileriahost interactions: which parasite molecules (proteins, lipids, RNA, other) are required for, or contribute to, these cancer-like phenotypes? Interactions with host signaling play a crucial role in transformation such as NF-kB. Size was a primary reason why this matter was unresolved. Theileria genomes contain about 4000 genes half of which still have no predicted function. According to Weir et al., (2010) two secreted multigene families SVSP (sub-telomere-encoded variable secreted protein) and TashAT (Theileria annulata schizont AT-hook protein) play a role in host-parasite interactions. The SVSP family is the largest gene family in both $T$. annulata and $T$. parva has been suspected of playing a role in immune evasion. TashAT-family proteins are secreted by the parasite which localize to the host nucleus and has been found to alter the expression and interfered DNA-binding domains in T. annulata. Theileria infection of bovine leukocytes modulates oncogenic signalling pathways such as JNK and AP-12. Peptidyl Prolyl Isomerase Pin1 (designated TaPin1) in T. annulata which is secreted into the host cell and modulates oncogenic signalling pathways. The TaPin1 is a prolyl isomerase and it interacts with the host ubiquitin ligase FBW7 leading to its degradation and subsequent stabilization of cJun which promotes transformation. Marsolier et al., (2015) conducted an in silico screening of parasite genome to identify proteins secreted by Theileria into the host cell and found that Theileria parasites secrete a prolyl isomerase to maintain host cell transformation. Theileria-induced replicative immortality, a very distinctive and enigmatic phenotype of Theileria transformed cells is that they can be cultured in vitro indefinitely, exactly as any standard established cell line. Theileria parasites invade lumphocytes using zippering mechanism and $\mathrm{PV}$ is lysed using roptries secretion. Parasite interferes with the NF B growth control by activating IKK complex. Moreover, Theileria parasite secretes prolyl isomerase to maintain host cell transformation. The parasite resides in the cytoplasm and associates with the host cells microtubules and centrosomes. When the host cell divides the parasite divides and seggregates alongside using host cells mitotic machenary, maintain an approximately $1: 1$ host-to-parasite ratio and the proliferation of infected cells is rapid and unchecked. Theileria schizont cause cancer like growth and transformation is parasite dependent but reversible. Moreover, many Theileria parasites do not seem to induce cancer hallmarks in their host cells and hence provide an excellent opportunity for comparative investigation. In the laboratory they provide a unique model to investigate how a simple eukaryotic cell can direct gene expression and induce proliferation of another complex eukaryotic host cell.

\section{References}

Ahmed JS, Hauschild S, Schein E. 1987. The role of interleukin 2 (IL 2) in the proliferation of Theileria annulata-infected bovine lymphocytes. Parasitol. res. 73(6): 524-6.

Campbel JD, Spooner RL. 1999. Macrophages behaving badly: infected cells and subversion of immune responses to Theileria annulata. Parasitology today. 15(1): 10-6.

Campbell JD, Nichani AK, Brown DJ, Howie SE, Spooner RL, Glass EJ. 1997. ParasiteMediated Steps in Immune Response Failure During Primary Theileria annulata infection. Trop. Ani. health prod. 29(4): 133S-5S.

Dobbelaere D, Heussler V. 1999. Transformation of leukocytes by Theileria parva and $T$. annulata. Annual Reviews in Microbiology. 53(1): 1-42.

Dobbelaere DA, Coquerelle TM, Roditi IJ, Eichhorn M, Williams RO. 1988. Theileria parva infection induces autocrine growth of 
bovine lymphocytes. Proceedings of the National Acad. of Sci. 85(13): 4730-4.

Dobbelaere DA, Coquerelle TM, Roditi IJ, Eichhorn M, Williams RO. 1988. Theileria parva infection induces autocrine growth of bovine lymphocytes. Proceedings of the National Academy of Sciences. 85(13): 4730-4.

Dobbelaere DA, Roditi IJ, Coquerelle TM, Kelke C, Eichhorn M, Williams RO. 1991. Lymphocytes infected with Theileria parva require both cell-cell contact and growth factor to proliferate. European j. immunol. (1): 89-95.

Duszenko M, Ginger ML, Brennand A, GualdrónLópez M, Colombo MI, Coombs GH, Coppens I, Jayabalasingham B, Langsley G, Lisboa de Castro S, Menna-Barreto R. 2011. Autophagy in protists. Autophagy. 7(2): $127-58$.

Glass E.J. 2001. The balance between protective immunity and pathogenesis in tropical theileriosis: what we need to know to design effective vaccines for the future. Res Vet. Sci. 70: 71-75.

Haller D, Mackiewicz M, Gerber S, Beyer D, Kullmann B, Schneider I, Ahmed JS, Seitzer U. 2010. Cytoplasmic sequestration of p53 promotes survival in leukocytes transformed by Theileria. Oncogene. (21): 3079.

Hayashida K, Kajino K, Hattori M, Wallace M, Morrison I, Greene MI, Sugimoto C. 2013. MDM2 regulates a novel form of incomplete neoplastic transformation of Theileria parva infected lymphocytes. Exp. Mol. patho. 94(1): 228-38.

Hooshmand-Rad P. 1976. The pathogenesis of anaemia in Theileria annulata infection. Res. in vet. sci. 20(3): 324-9.

Hulliger L, Brown CG, Wilde JK. 1966. Transition of developmental stages of Theileria parva in vitro at high temperature. Nature. 211(5046): 328.

Hulliger L, Wilde JK, Brown CG, Turner L. 1964. Mode of multiplication of Theileria in cultures of bovine lymphocytic cells. Nature. 203(4946): 728.

Jensen K, Makins GD, Kaliszewska A, Hulme MJ, Paxton E, Glass EJ. 2009. The protozoan parasite Theileria annulata alters the differentiation state of the infected macrophage and suppresses musculoaponeurotic fibrosarcoma oncogene (MAF) transcription factors. International journal for parasitol. 39(10): 1099-108.

Levine, A.J., 1997. p53, the cellular gatekeeper for growth and division. cell, 88(3), pp. 323-331.

Marsolier J, Perichon M, DeBarry JD, Villoutreix BO, Chluba J, Lopez T, Garrido C, Zhou XZ, Lu KP, Fritsch L, Ait-Si-Ali S. 2015. Theileria parasites secrete a prolyl isomerase to maintain host leukocyte transformation. Nature. 520 (7547): 378.

Metheni M, Echebli N, Chaussepied M, Ransy C, Chéreau C, Jensen K, Glass E, Batteux F, Bouillaud F, Langsley G. 2014. The level of $\mathrm{H}_{2} \mathrm{O}_{2}$ type oxidative stress regulates virulence of Theileria-transformed leukocytes. Cellular microbiology. 16(2): 269-79.

Nichani AK, Craigmile SC, Spooner RL, Campbell JD. 1999. Diminished IL-2 responses and alteration of CD2 expression on $\mathrm{CD} 8+\mathrm{T}$ cells are associated with a lack of cytotoxic $\mathrm{T}$ cell responses during Theileria annulata infection. Cli. Exp immunol. (2): 316.

Preston, P. M. 2001. The Encylopedia of Arthopode Transmitted Infections, Ist Ed. CABI publishing, Wallingford (UK) pp. 487-504.

Preston, P. M., Hall, F. R., Glass, E.J., Campbell, J.D., Darghouth, M. A., Ahmad, J. S., Shiels, B.R., Spooner, R. L., Jongejan, F., Brown, C. G. 1999. Inate and adaptive immune responses co-operate to protect cattle against Theileria annulata. Parasitol Today. 15: 268-74.

Reddel RR. 2000. The role of senescence and immortalization in carcinogenesis. Carcinogenesis. 21(3): 477-84.

Schmuckli-Maurer J, Casanova C, Schmied S, Affentranger S, Parvanova I, Kang'a S, Nene V, Katzer F, McKeever D, Müller J, Bishop R. 2009. Expression analysis of the Theileria parva subtelomere-encoded variable secreted protein gene family. PloS one. 4(3):e4839. 
Schnittger L, Katzer F, Biermann R, Shayan P, Boguslawski K, McKellar S, Beyer D, Shiels BR, Ahmed JS. 2000. Characterization of a polymorphic Theileria annulata surface protein (TaSP) closely related to PIM of Theileria parva: implications for use in diagnostic tests and subunit vaccines. Molecular and biochemical parasitol. 120(2): 247-56.

Seluanov A, Hine C, Azpurua J, Feigenson M, Bozzella M, Mao Z, Catania KC, Gorbunova V. 2009. Hypersensitivity to contact inhibition provides a clue to cancer resistance of naked mole-rat. Proceedings of the National Academy of Science. 106(46): 19352-7.

Shaw MK.1997. The same but different: the biology of Theileria sporozoite entry into bovine cells. International journal for parasitol.1; 27(5): 457-74.

Shayan P, Schop B, Conze G, Schein E, Ahmed JS. 1999. Is interleukin 2 necessary for the autocrine proliferation of Theileria-infected bovine cells?. Parasitol. res. 85(5): 409-12.

Shiels B, Kinnaird J, McKellar S, Dickson J, Miled LB, Melrose R, Brown D, Tait A. 1992. Disruption of synchrony between parasite growth and host cell division is a determinant of differentiation to the merozoite in Theileria annulata. J. of Cell Sci. 101(1): 99-107.

Shiels B, Kinnaird J, McKellar S, Dickson J, Miled LB, Melrose R, Brown D, Tait A. 1992. Disruption of synchrony between parasite growth and host cell division is a determinant of differentiation to the merozoite in Theileria annulata. J. of Cell Sci. 101(1): 99-107.

Shiels B, Langsley G, Weir W, Pain A, McKellar S, Dobbelaere D.2006. Alteration of host cell phenotype by Theileria annulata and Theileria parva: mining for manipulators in the parasite genomes. International journal for parasitol. 36(1):9-21.

Tait A, Hall FR. 1990. Theileria annulata: control measures, diagnosis and the potential use of subunit vaccines. Rev Sci Tech. (2): 387403.

Vogelstein B, Papadopoulos N, Velculescu VE, Zhou S, Diaz LA, Kinzler KW. 2013. Cancer genome landscapes. science. 339(6127): 1546-58.

Von Schubert C, Xue G, Schmuckli-Maurer J, Woods KL, Nigg EA, Dobbelaere DA. 2010. The transforming parasite Theileria co-opts host cell mitotic and central spindles to persist in continuously dividing cells. PLoS biology. 28; 8(9): e1000499.

Weir W, Karagenç T, Baird M, Tait A, Shiels BR. 2010. Evolution and diversity of secretome genes in the apicomplexan parasite Theileria annulata. BMC genomics. 11(1): 1.

Weitzman, M.D. and Weitzman, J.B. 2014. What's the damage? The impact of pathogens on pathways that maintain host genome integrity. Cell Host Microbe 15, 283-294

Woods KL, Theiler R, Mühlemann M, Segiser A, Huber S, Ansari HR, Pain A, Dobbelaere DA. 2013. Recruitment of EB1, a master regulator of microtubule dynamics, to the surface of the Theileria annulata schizont. PLoS pathogens. 9(5): e1003346.

Zhou F. 2009. Molecular mechanisms of IFN- $\gamma$ to up-regulate MHC class I antigen processing and presentation. Int. reviews immunol. 28(3-4): 239-60.

\section{How to cite this article:}

Aquil Mohmad, Bilal Ahmad Malla, Ishfaq Maqbool, Zahoor Ahmad War, Irshad Ahmad Para and Sheikh Firdous Ahmad. 2018. Theileria-Induced Leukocyte Transformation: A Parasite Model for the Study of Lympho Proliferation. Int.J.Curr.Microbiol.App.Sci. 7(11): 2814-2821. doi: https://doi.org/10.20546/ijcmas.2018.711.324 